\title{
Estrategia metodológica para la actividad investigadora de la Brigada Médica cubana en Guatemala
}

\author{
Mabel Rocha-Vázquez
}

Introducción. La presentación de trabajos científicos en eventos no siempre se distingue por la calidad y responde a veces a la mera necesidad de hacerlo por el hecho que es evaluado.

objetivo. Describir las principales deficiencias de los informes de investigación presentados y proponer una estrategia para su solución.

Materiales y métodos. Estudio descriptivo, retrospectivo, con diseño cuanticualitativo para lo cual se revisaron las 105 planillas de evaluación elaboradas por los tribunales del evento de base del año 2012 y las 105 relatorías de los tribunales sobre cada trabajo presentado.

Resultados. Se detectaron deficiencias en la evaluación tanto de pósters como de temas libres, lográndose como promedio general sólo el $87,40 \%$ y el $88,03 \%$ del total de puntos a obtener, respectivamente. En los pósters se identificaron las mayores dificultades en aspectos metodológicos del contenido y aspectos formales, y en los temas libres, en el informe escrito con cumplimiento de aspectos metodológicos. Destacan con mayor repercusión la formulación correcta de objetivos, la calidad de gráficos y tablas, el diseño metodológico correcto, la calidad científica y el análisis estadístico.

Conclusiones. Se evidencian múltiples deficiencias metodológicas a las cuales responde la estrategia metodológica propuesta, que aborda de forma integral la investigación científica como proyecto, como proceso y sus resultados.

Palabras clave. Deficiencias. Estrategia metodológica. Investigaciones científicas.

\section{Methodological strategy for research activity in the Cuban Medical Brigade in Guatemala}

Introduction. The present scientific papers at events not always distinguished by quality and sometimes responds to the mere need for it by the fact that it is evaluated.

Aim. To describe the main shortcomings of the research reports presented and propose a strategy for resolution.

Materials and methods. A descriptive, retrospective design for which reviewed all of the evaluation forms developed by the courts based event in 2012 and review of $100 \%$ of the rapporteur of the courts of each work presented.

Results. We identified weaknesses in the assessment of both poster as free themes, achieving overall average only $87.40 \%$ and $88.03 \%$ of total points obtained respectively. In the poster we identified the major difficulties in methodological aspects of content and formal aspects and free items in the written report compliance with methodological aspects, highlighting the impact more correct formulation of objectives and the quality of graphics and tables together to design correct methodological and scientific quality and statistical analysis.

Conclusions. We showed multiple methodological deficiencies which respond to the methodological proposal comprehensively addresses scientific research as a project, as a process and its results.

Key words. Gaps. Methodological strategy. Scientific research.

\section{Introducción}

La preocupación y búsqueda de la excelencia académica ha sido una constante en la historia de las instituciones de educación superior. El concepto de calidad, de representar el control que se hace al final del proceso, ha pasado a ser la evaluación que se realiza de todas las actividades, que sistematizadas y organizadas constituyen el aseguramiento de la calidad [1].

La medicina es una ciencia de probabilidades y un arte de manejar la incertidumbre; por ello, buscar respuestas a dicha incertidumbre es una fuente inagotable de investigación $[2,3]$.
Universidad de Ciencias Médicas de Cienfuegos. Cienfuegos, Cuba.

Correspondencia: Dra. Mabel Rocha Vázquez. Universidad de Ciencias Médicas de Cienfuegos. Edif. 19, piso 16, apartamento 1601. CP 55100 Pastorita Cienfuegos, Cuba.

E-mail: mabelrochavzquez@yahoo.es

Conflicto de intereses: No declarado.

Competing interests: None declared.

(c) $2015 \mathrm{FEM}$ 
Tabla I. Presentación de pósters: puntuación obtenida por aspectos.

\begin{tabular}{|c|c|c|c|}
\hline & $\begin{array}{c}\text { Total de } \\
\text { posibles puntos } \\
\text { a obtener }\end{array}$ & $\begin{array}{l}\text { Promedio } \\
\text { de puntos } \\
\text { obtenidos }\end{array}$ & $\begin{array}{c}\% \text { del } \\
\text { total }\end{array}$ \\
\hline Aspectos metodológicos del contenido & 50 & 43,23 & 86,47 \\
\hline Título correcto & 5 & 4,74 & 94,88 \\
\hline Breve introducción y formular objetivo general & 15 & 12,44 & 82,95 \\
\hline Valor de los resultados del trabajo & 20 & 16,98 & 84,88 \\
\hline Aporte al trabajo de la brigada & 10 & 9,07 & 90,70 \\
\hline Aspectos formales & 35 & 30,33 & 86,64 \\
\hline Originalidad en la presentación & 10 & 8,60 & 86,05 \\
\hline Cumplimiento de aspectos de presentación impresa & 25 & 21,72 & 86,88 \\
\hline Uso adecuado de letras & 5 & 4,40 & 87,91 \\
\hline Uso adecuado del espacio del póster & 5 & 4,63 & 92,56 \\
\hline Uso adecuado de ortografía y redacción & 5 & 4,70 & 93,95 \\
\hline Presentación correcta de resultados & 10 & 8,00 & 80,00 \\
\hline Presentación en sesión del póster & 15 & 13,84 & 92,25 \\
\hline Calidad de la breve exposición ante el tribunal & 8 & 7,40 & 92,44 \\
\hline Respuestas a preguntas del tribunal o participantes & 7 & 6,44 & 92,03 \\
\hline Total & 100 & 87,40 & 87,40 \\
\hline
\end{tabular}

El profesional de la salud y específicamente el médico, ante cada paciente que acude en busca de ayuda, desde que comienza su interrogatorio está estrechamente vinculado al proceso de investigación, y este proceso requiere preparación [3-5].

A pesar de ser una necesidad, varios autores coinciden en señalar que existen dificultades importantes para la realización de investigación [1,2,4-12].

La especialización médica en Cuba garantiza a los residentes una sólida formación; el residente no sólo aprende de forma independiente en la práctica de su profesión, sino que aplica su creatividad y criterio científico a la solución de cada problema de la práctica médica [1].

Sin embargo, ¿están realmente preparados nuestros profesionales, desde el punto de vista teórico y práctico, para enfrentarse a la investigación científica que demanda su profesión? Ésta podría ser una arista del problema que evidencie deficiencias en este proceso que generen la necesidad de diseñar acciones correctivas para incrementar la calidad del proceso investigador.

\section{Materiales y métodos}

Investigación con diseño cuanticualitativo, descriptiva y retrospectiva, llevada a cabo en noviembre del 2012 en la Brigada Médica cubana en Guatemala.

De 22 jornadas de base a realizar, se seleccionaron de forma intencional 12 (54,54\%), todas las realizadas hasta el momento en que se comenzó el estudio. Las jornadas de base son aquellas que se realizan en cada uno de los 22 departamentos donde la Brigada Médica cubana presta sus servicios en el país, en las cuales los profesionales presentan los resultados de las investigaciones científicas realizadas en el período.

Se revisaron los 105 trabajos presentados en tema libre y póster en las jornadas seleccionadas y las 105 relatorías de tribunales sobre dichos trabajos.

Se realizó una revisión de las planillas de evaluación, para lo cual se elaboró un instructivo, buscando homogeneidad en los criterios. De forma general, para la puntuación se tuvo en consideración la novedad y actualidad del tema investigado, aspectos metodológicos del trabajo y la presentación ante el tribunal, que incluye su defensa ante preguntas formuladas. La puntuación particular de cada aspecto se incluye en las tablas. La puntuación obtenida en cada uno de los aspectos se lleva al porcentaje que representa del total de puntos a obtener, con el fin de facilitar la interpretación de los resultados.

Respecto a las relatorías de tribunales, se realizó un análisis de contenido y los resultados se presentan expresando entre paréntesis el número de veces que se repite una idea.

\section{Resultados}

\section{Presentación de pósters}

Como se refleja en la tabla I, en los pósters presentados, de forma general se logra obtener como media el $87,40 \%$ del total de puntos. Solamente la presentación en sesión de pósters propiamente dicha supera el $90 \%$ del total de puntos a obtener. Dentro de los aspectos metodológicos del contenido superan el $90 \%$ del total de puntos a obtener el título correcto y el aporte al trabajo. El aspecto con mayores dificultades resultó ser la presentación correcta de los resultados.

\section{Presentación de temas libres}

En la tabla II se muestran los resultados obtenidos en la presentación de temas libres; la media de puntos 
obtenidos fue del 88,03\% del total. Resultó ser la presentación del trabajo ante el tribunal el que logró el mayor promedio de puntos, seguido del aporte a la solución de problemas. Por su parte, el cumplimiento de los aspectos metodológicos en el informe escrito alcanza el $85 \%$ del total de puntos, con menores puntuaciones en formulación correcta de objetivos y calidad de gráficos y tablas.

\section{Revisión de la relatoría de los tribunales}

- Necesidad de ser generalizado (12).

- Debe mejorar la calidad de gráficos y tablas (7).

- Dificultades en las referencias bibliográficas (7).

- Presentación de póster con deficiencias en el formato (6).

- La introducción no aborda en profundidad el tema investigado (5).

- Errores ortográficos (5).

- Dificultades en la formulación de los objetivos (5).

- El título del trabajo no expresa su contenido (3).

- Dificultades en la defensa del trabajo (1).

\section{Descripción de la estrategia metodológica propuesta}

Se organiza abordando la investigación como proyecto, como proceso y sus resultados; se definen tareas para cada una de ellas con responsables, participantes, fecha de cumplimiento e indicadores de evaluación. Enunciamos a continuación las tareas:

\section{Investigación como proyecto}

- Definir líneas de investigación en función de las prioridades.

- Diseñar el formato de presentación de los manuscritos.

- Establecer criterios para la evaluación.

- Definir criterios para otorgar premios.

- Definir el sistema estadístico para informar de los resultados.

- Capacitar a organizadores y ejecutores del proceso.

- Capacitar en metodología de la investigación según necesidades.

\section{Investigación como proceso}

- Divulgar la convocatoria de eventos.

- Asignar investigaciones por encargo según necesidades.

- Evaluar proyectos por comisiones y seguimiento.

- Conformar comisiones organizadoras de eventos de base.

- Seleccionar y preparar tribunales del evento.

- Reconocer los resultados obtenidos de forma in-
Tabla II. Presentación de temas libres: puntuación obtenida por aspectos.

\begin{tabular}{|c|c|c|c|}
\hline & $\begin{array}{c}\text { Total de } \\
\text { posibles puntos } \\
\text { a obtener }\end{array}$ & $\begin{array}{l}\text { Promedio } \\
\text { de puntos } \\
\text { obtenidos }\end{array}$ & $\begin{array}{l}\% \text { del } \\
\text { total }\end{array}$ \\
\hline Novedad y actualidad del tema & 10 & 9,21 & 92,10 \\
\hline Informe escrito: cumplimiento de los aspectos metodológicos & 60 & 51,08 & 85,13 \\
\hline Título & 5 & 4,61 & 92,26 \\
\hline Resumen & 5 & 4,27 & 85,48 \\
\hline Introducción y enfoque del problema a investigar & 5 & 4,53 & 90,65 \\
\hline Formulación correcta de los objetivos & 5 & 3,85 & 77,10 \\
\hline Diseño metodológico correcto y de calidad científica & 10 & 8,21 & 82,10 \\
\hline Análisis estadístico adecuado & 5 & 4,03 & 80,65 \\
\hline Calidad de los gráficos y tablas & 5 & 3,95 & 79,03 \\
\hline Discusión adecuado de los resultados & 10 & 8,98 & 89,84 \\
\hline Conclusiones & 5 & 4,31 & 86,13 \\
\hline Referencias bibliográficas & 5 & 4,32 & 86,45 \\
\hline $\begin{array}{l}\text { Aporte a la solución de problemas } \\
\text { del país y de la Brigada Médica cubana }\end{array}$ & 10 & 9,03 & 90,32 \\
\hline Presentación del trabajo ante el tribunal & 20 & 18,71 & 93,55 \\
\hline Claridad en la exposición, dicción & 2 & 1,84 & 91,94 \\
\hline Ajuste al tiempo & 3 & 2,42 & 80,65 \\
\hline Uso adecuado de medios auxiliares & 3 & 2,79 & 93,01 \\
\hline Dominio del tema & 4 & 3,90 & 97,58 \\
\hline Coherencia en la exposición & 3 & 2,89 & 96,24 \\
\hline Defensa ante preguntas del tribunal o del auditorio & 5 & 4,87 & 97,42 \\
\hline Total general & 100 & 88,03 & 88,03 \\
\hline
\end{tabular}

dividual a los autores y de forma colectiva a las brigadas destacadas.

- Emitir información del resumen según se determine.

\section{Investigación como resultado}

- Consolidar y divulgar el resumen (cuanticualitativamente).

- Utilizar la información anterior como mecanismo de retroalimentación que incremente la calidad.

- Desarrollar el evento de generalización de resultados.

- Divulgar resultados de investigaciones presentadas, que incluye la publicación de éstos.

- Comparar los resultados con eventos anteriores. 


\section{Discusión}

Estudios realizados por Romeu-Escobar et al [4] plantean como principales deficiencias: problema científico no bien definido; deficiencias en la elaboración de objetivos; escasa novedad científica; aplicabilidad y grado de generalización; deficiencias en la explicación de las variables, métodos, técnicas y procedimientos, y falta de calidad en el análisis y la discusión de los resultados. Muchos de estos aspectos coinciden con nuestros hallazgos y con los de otros autores $[1,5]$.

En 12 tesis revisadas por Sarasa-Muñoz et al [1], aunque algunos de los objetivos específicos declarados daban salida a diferentes aspectos del problema, otros constituían tareas de la investigación, resultados que coinciden con los nuestros.

Por su parte, Austin plantea que los términos utilizados para definir el problema deben ser lo bastante claros como para permitir que cualquier persona, con sólo leerlo, se ubique en lo que se pretende estudiar; añade que delimitar los aspectos que abarca el problema evita divagaciones porque permite que el investigador centre la atención en los elementos medulares del problema científico [1].

Preocupan en particular las dificultades en concretar un problema científico: si no se ha definido bien el problema, toda la investigación carece de rigor y probablemente sea a partir de este elemento que se hayan derivado las otras insuficiencias que se identificaron.

La realización de investigaciones con escasa novedad científica coincide también con otros autores $[3,4]$.

En otros estudios se identificaron conclusiones que no respondían directamente a los objetivos propuestos, lo cual coincide con nuestro resultados $[1,3,5]$.

Alonso-Moreno asegura que, para investigar, es fundamental el apoyo metodológico y la sistematización en la investigación. En 20 trabajos revisados (54,05\%) encontró una pobre contextualización y argumentación de la problemática en la introducción; en ninguno de los trabajos quedó clara la delimitación y progresividad del problema, y en 16 trabajos $(43,24 \%)$ faltaron aspectos teóricos esenciales de la temática abordada. En varios trabajos existían también deficiencias en la denominación de las tablas $[1,5]$, resultados muy similares a los obtenidos en la presente investigación.

Otros autores reconocen dificultades en la forma de acotar la bibliografía consultada y en la calidad general de ésta [3-5].
La necesidad de proponer estrategias de gestión del conocimiento para resolver problemas similares fue detectada en otras investigaciones [5,6,8-12].

En correspondencia con la bibliografía revisada, la estrategia que proponemos parte de un objetivo general, seguido de un plan estratégico en el que se precisan las etapas y acciones, y cómo deben instrumentarse y evaluarse cada una de ellas $[6,7]$.

\section{Bibliografía}

1. Sarasa Muñoz-NL, Cañizares-Luna O, Yedra-Díaz AM, Ríos-Vega LE, Santana-Fonseca EC. Deficiencias del proceso formativo de investigaciones de los residentes de medicina general integral. Estado Zulia. Curso 2008-2009. PortalesMedicos. com; 2010. URL: http://www.portalesmedicos.com/ publicaciones/articles/2151/1/Deficiencias-del-procesoformativo-de-investigacion-de-los-residentes-de-MedicinaGeneral-Integral.html. [18.11.2012].

2. Pita-Fernández S, Pértega-Díaz S. Dificultades de la investigación en atención primaria. Fisterra.com; 2005. URL: http://www. fisterra.com $/ \mathrm{mbe} /$ investiga/difInvestAP/difInvesAP.asp. [08.11.2012].

3. Jorge-Fernández M, Rubio-Olivares DY, González-Sánchez R, Fundora-Mirabal J, Castellanos-Laviña JC, Curbelo-Menéndez O. La formación investigativa de los estudiantes de medicina. Biblioteca Virtual en Salud de Cuba; 2008. URL: http://bvs. sld.cu/revistas/ems/vol22_4_08/ems05408.htm. [18.11.2012].

4. Romeu-Escobar M, Sabina-Romeu B, Almaguer-Sabina P, Lara-Calderín G. Las investigaciones científicas en medicina general integral durante la evolución histórica de la especialidad. Cienfuegos, 1988-2005. MediSur 2006; 4: 420-6. URL: http:// www.redalyc.org/articulo.oa?id=180019873006. [28.10.2012].

5. Ramírez C, Arellano L, Morales A, Fernández H, Pineda E, Quintero Y. Planeación estratégica. Henry Mintzberg. URL: http://www.slideshare.net/jcfdezmxestra/planeacin-estratgicahenry-mintzberg. [21.11.2012].

6. Borges-Obregón D, Padrón-Pereira E, Negrín-Pérez RI. Estrategia metodológica para el perfeccionamiento de la enseñanza-aprendizaje de los bailes populares cubanos en los estudiantes de la carrera de Cultura Física. Efdeportes.com; 2009. URL: http://www.efdeportes.com/efd128/ensenanzaaprendizaje-de-los-bailes-populares-cubanos.htm. [08.11.2012].

7. Montes de Oca-Recio N, Machado-Ramírez EF. Estrategias docentes y métodos de enseñanza-aprendizaje en la educación superior. Rev Hum Med; 2011. URL: http://scielo.sld.cu/ scielo.php?pid=S1727-81202011000300005\&script $=$ sci arttext. [20.11.2012].

8. Vega-Cubillo E, García-Sánchez JA, García-Ortega P, Carvajal A. La investigación en atención primaria debe ser potenciada. Aten Primaria 2002; 30: 97.

9. Bayarre-Vea HD, Pérez-Piñero JS, Couturejuzón-González L, Sarduy-Domínguez Y, Castañeda-Abascal IE, Díaz-Llanes G. La formación avanzada de investigadores en el ámbito de la atención primaria de salud, una necesidad impostergable. Rev Cubana Med Gen Integr; 2009. URL: http://www.bvs. sld.cu/revistas/mgi/vol25_2_09/mgi15209.htm. [25.03.2010].

10. Jowett SM, Macleod J, Wilson S, Hobbs FD. Research in primary care: extent of involvement and perceived determinants among practitioners from one English region. Br J Gen Pract 2000; 50: 387-9.

11. Falk WA. Research in general practice. Can Med Assoc J 1979; 120: 1198-200.

12. Fernández de Sanmamed MJ, De la Fuente JA, Mercader J, Borrel C, Martín C, Birulés M, et al. Factores relacionados con la actividad investigadora de los médicos de atención primaria. Aten Primaria 1997; 19: 283-9. 